\title{
Circ_0072083 interference enhances growth-inhibiting effects of cisplatin in non-small-cell lung cancer cells via miR-545-3p/CBLL1 axis
}

Huiping Li, Fangfang Liu and Wenjing Qin * (1)

\begin{abstract}
Background: Non-small-cell lung cancer (NSCLC) is one of the common cancers in the world. Circular RNA 0072083 (circ_0072083, circZFR) has been reported to be associated with the progression of NSCLC. In this study, we intended to explore the role and the potential mechanism of circ_0072083 in NSCLC.

Methods: Quantitative real time polymerase chain reaction (qRT-PCR) was performed to detect the expression of circ_0072083, its matching linear RNA (zinc finger RNA binding protein (ZFR)) and microRNA-545-3p (miR-545-3p) in NSCLC cells. The ability of colony formation in NSCLC cells was detected by colony formation assay. The apoptosis and cell cycle were measured by flow cytometry. The metastasis was determined by transwell migration and invasion assays. The protein expression of E-cadherin, $\mathrm{N}$-cadherin, Vimentin and Cbl proto-oncogene like 1 (CBLL1) was examined by western blot assay. The interaction between miR-545-3p and circ_0072083 or CBLL1 was predicted by starBase or Targetscan software. Dual-luciferase reporter assay and RNA immunoprecipitation (RIP) assay were applied to validate these interactions. Nude mice bearing tumors were used to confirm the role of circ_0072083 and cisplatin (DDP) in vivo.
\end{abstract}

Results: The level of circ_0072083 was higher in NSCLC tissues and cells relative to that in adjacent non-tumor tissues and normal lung cells. The transfection of si-circ_0072083 inhibited colony formation, cell cycle and metastasis while promoted the apoptosis of NSCLC cells stimulated by DDP. MiR-545-3p was a direct functional target of circ_0072083 in NSCLC cells. CBLL1 could bind to miR-545-3p in NSCLC cells. Circ_0072083 promoted the progression of NSCLC induced by DDP through sponging miR-545-3p and enhancing the enrichment of CBLL1 in vivo and in vitro. Conclusion: Circ_0072083 depletion contributed to DDP-triggered inhibition of NSCLC tumor through miR-545-3p/ CBLL1 axis.

Keywords: NSCLC, circ_0072083, miR-545-3p, CBLL1, DDP

*Correspondence: qinwenjing007@163.com

Department of Respiratory Medicine, First Affiliated Hospital of Henan University, No. 357, Ximen Street, Longting District, Kaifeng 475000, Henan, China

\section{Background}

Lung cancer is the leading cause of the cancer-associated mortality globally $[1,2]$. There were 2093,876 new cases and 1,761,007 deaths of lung cancer globally in 2018 , accounting for $11.6 \%$ and $18.4 \%$ of all cancers, respectively [2]. Lung cancer contains small-cell lung cancer (SCLC) and non-small-cell lung cancer

(c) The Author(s) 2020. This article is licensed under a Creative Commons Attribution 4.0 International License, which permits use, sharing, adaptation, distribution and reproduction in any medium or format, as long as you give appropriate credit to the original author(s) and the source, provide a link to the Creative Commons licence, and indicate if changes were made. The images or other third party material in this article are included in the article's Creative Commons licence, unless indicated otherwise in a credit line to the material. If material is not included in the article's Creative Commons licence and your intended use is not permitted by statutory regulation or exceeds the permitted use, you will need to obtain permission directly from the copyright holder. To view a copy of this licence, visit http://creativeco mmons.org/licenses/by/4.0/. The Creative Commons Public Domain Dedication waiver (http://creativecommons.org/publicdomain/ zero/1.0/) applies to the data made available in this article, unless otherwise stated in a 
(NSCLC). Among the two types of lung cancer, NSCLC accounts for $85 \%$ [3]. The therapeutic methods for NSCLC patients include surgery resection, chemotherapy, radiotherapy, targeted therapy and immunotherapy [4]. In the case of chemotherapy, the chemoresistance of NSCLC patients remains a big obstacle for the curing the NSCLC. Therefore, uncovering the resistance mechanism of NSCLC cells is urgent to avoid the metastasis or neoplasm recurrence.

Circular RNAs (circRNAs) are a class of non-coding RNAs, and they possess the closed loop structure [5]. CircRNAs are crucial modulators in cancers [6-8]. Circ_0072083 (circZFR) could regulate the progression of multiple cancers through acting as microRNAs (miRNAs) sponges. Wei et al. proved that circ_0072083 promoted the development of papillary thyroid cancer through miR-1261/C8orf4 axis [9]. Tan et al. reported that circ_0072083 contributed to the progression of hepatocellular cancer via miR-3619-5p/CTNNB1 signaling [10]. Wang et al. demonstrated that circ_0072083 accelerated the proliferation and metastasis of human renal carcinoma via sponging miR-206 [11]. Zhang et al. found that circ_0072083 acted as an oncogene in NSCLC via miR-101-3p/CUL4B axis [12]. Nevertheless, the working network of circ_0072083 in NSCLC remains not fully elucidated.

MiRNAs have emerged as pivotal regulators in cancers. Muhammad et al. found that miR-203 served as an oncogene in ER-positive breast cancer, and it accelerated the growth and stemness of ER-positive breast cancer via SOCS3 [13]. Fan et al. demonstrated that miR-125a impeded the proliferation and motility of cervical cancer cells through suppressing STAT3 [14]. Du et al. reported that miR-33a inhibited the proliferation of NSCLC cells through METTL3 [15]. Wang et al. proved that miR204 repressed the motility of NSCLC cells via JAK2 [16]. Besides, miR- 545 has been reported to hamper the progression of NSCLC via ZEB2 [17]. However, the interaction between circ_0072083 and miR-545-3p in NSCLC has not been reported.

Cbl proto-oncogene like 1 (CBLL1) is an E3 ubiquitin ligase with RING-finger domain. Accumulating articles have reported the role of CBLL1 in NSCLC. Hui et al. found that the expression of CBLL1 was higher in NSCLC, and it facilitated the growth and motility of NSCLC cells [18]. Qui et al. reported that XIST accelerated the proliferation and metastasis of NSCLC cells via acting as a miR-212-3p sponge and up-regulating CBLL1 [19]. In the present study, we investigated whether miR545-3p exerted its function through CBLL1 in NSCLC.

We measured the abundance of circ_0072083, miR545-3p and CBLL1 in NSCLC tissues and cells. Moreover, we illustrated the mechanism by which circ_0072083 contributed to the chemoresistance of NSCLC cells to cisplatin (DDP).

\section{Materials and methods Clinical samples}

The NSCLC tumor tissues and adjacent non-tumor tissues were acquired from a total of 31 NSCLC patients in First Affiliated Hospital of Henan University. Written informed consents were obtained from all subjects in this study. The utilization of human material was approved by the Institute Research Ethics Committee of First Affiliated Hospital of Henan University.

\section{Cell culture and DDP treatment}

Human NSCLC cell lines (H522 and A549) and normal human lung epithelial cell line BEAS-2B were purchased from BeNa Culture Collection (Beijing, China) and cultivated in Dulbecco's Modified Eagle's Medium (DMEM, Gibco, Carlsbad, CA, USA) added with $10 \%$ heat-inactivated fetal bovine serum (FBS, Gibco), 100 units $/ \mathrm{mL}$ penicillin and $100 \mu \mathrm{g} / \mathrm{mL}$ streptomycin under $37{ }^{\circ} \mathrm{C}$ humidified incubator with $5 \% \mathrm{CO}_{2} .30 \mu \mathrm{g} / \mathrm{mL}$ DDP was used to stimulate NSCLC cells for $48 \mathrm{~h}$.

\section{Quantitative real-time polymerase chain reaction (qRT-PCR)}

qRT-PCR was conducted to detect the enrichment of relevant RNAs. RNA sample was isolated with TRIzol (Beyotime, Shanghai, China). Reverse transcription was conducted using High-Capacity cDNA Reverse Transcription Kit (Applied Biosystems, Foster City, CA, USA). Hieff Unicon aqMan multiplex qPCR master mix (YEASEN, Shanghai, China) was used to conduct qRTPCR reaction. Glyceraldehyde-3-phosphate dehydrogenase (GAPDH) or U6 was acted as the internal controls. The abundance of RNA was calculated using the formula of $2^{-\Delta \Delta C t}$. The primer sequences were listed as below: circ_0072083, Forward primer (FP), 5'-AACCAC CACAGATTCACTAT-3', Reverse primer (RP), 5'-AAC CACCACAGATTCACTAT-3'; zinc finger RNA binding protein (ZFR; circ_0072083 matching linear RNA), FP, 5'-GCCCAGCCGGCTGGGGGAAG-3', RP, 5'-GCA GCTACTGGAGCCTGATG-3'; miR-545-3p, FP, 5'-TGG CTCAGTTCAGCAGGAAC- $3^{\prime}$, RP, universal reverse primer; GAPDH, FP, 5'-ATAGCACAGCCTGGATAG CAACGTAC- $3^{\prime}, \quad \mathrm{RP}, \quad 5^{\prime}$-CACCTTCTACAATGAGCT GCGTGTG-3'; U6, FP, 5'-CGCTTCGGCAGCACATAT AC-3', RP, 5'-TTCACGAATTTGCGTGTCAT-3'.

\section{RNase R treatment}

To confirm the loop structure of circ_0072083, RNase $\mathrm{R}$ was used in this study. $40 \mathrm{U}$ RNase $\mathrm{R}$ was incubated with $10 \mu \mathrm{g}$ RNA sample for $15 \mathrm{~min}$, and the abundance of 
circ_0072083 and ZFR was determined through performing qRT-PCR assay.

\section{Cell transfection}

NSCLC cells were plated in 6-well plates and transfected with the following RNA or plasmid with lipofectamine 3000 (Invitrogen, Carlsbad, CA, USA). Circ_0072083 specific small interfering RNA (si-circ_0072083), siRNA negative control (si-NC), circ_0072083 specific short hairpin RNA (Sh-circ_0072083) and its control, miR545-3p mimic (miR-545-3p), miR-NC, miR-545-3p inhibitor (anti-miR-545-3p) and its matching control (anti-miR-NC), CBLL1 plasmid (CBLL1) and its matching empty vector (vector) were obtained from Genepharma (Shanghai, China).

\section{Colony formation assay}

A549 or H522 cells were transferred into 6-well plates at a concentration of 150 cells/well. The cells were cultured for 2 weeks until the cell colonies were visible, and the culture medium was replaced with fresh medium every 3 days. The colonies were immobilized and then stained using crystal violet.

\section{The apoptosis detection by flow cytometry}

Annexin-V-fluorescein isothiocyanate (FITC) Apoptosis Detection Kit (R\&D Systems, Abingdon, UK) was utilized to detect the apoptosis of NSCLC cells. Briefly, NSCLC cells were collected and then re-suspended in $100 \mu \mathrm{L}$ binding buffer. Annexin-V-FITC and propidium iodide (PI) were incubated with NSCLC cells in the dark for $15 \mathrm{~min}$. The apoptosis of NSCLC cells was analyzed on FC-500 flow cytometer (Beckman Coulter, Pasadena, CA, USA).

\section{Cell cycle detection by flow cytometry}

NSCLC cells were immobilized with $70 \%$ ethanol at $-20^{\circ} \mathrm{C}$ overnight. Subsequently, the cells were incubated with RNase for $30 \mathrm{~min}$ at $37^{\circ} \mathrm{C}$ to remove the RNA. PI was added to the cells to stain the DNA at $4{ }^{\circ} \mathrm{C}$ for $1 \mathrm{~h}$. FC-500 flow cytometer (Beckman Coulter) was used to detect the cell cycle.

\section{Transwell migration and invasion assays}

Transwell chambers were purchased from Millipore (Billerica, MA, USA). To detect the migration of NSCLC cells, A549 and H522 cells in serum-free medium were transferred into the upper chambers with the noncoated membrane. To detect the invasion of NSCLC cells, the upper chambers were pre-coated with $100 \mu \mathrm{L}$ Matrigel (BD Biosciences, San Jose, CA, USA). 10\% FBS was added into the lower chambers to act as chemotactic factor. The migrated or invaded cells in the lower face of the membrane were immobilized and then dyed with $1 \%$ crystal violet. The cell numbers in three random fields were counted using an optical microscope.

\section{Western blot assay}

H522 and A549 cells were harvested and lysed using cell lysis buffer (Beyotime). The concentration of protein samples was determined using bicinchoninic acid (BCA). A total of $30 \mu \mathrm{g}$ protein sample was separated using $10 \%$ sodium dodecyl sulfate polyacrylamide gel electrophoresis (SDS-PAGE) and transferred into polyvinylidene difluoride (PVDF) membranes (Millipore). The membranes were blocked using $5 \%$ skim milk for $1 \mathrm{~h}$, and then the membranes were probed with the following antibodies at $4{ }^{\circ} \mathrm{C}$ overnight: anti-E-cadherin (ab40772, Abcam, Cambridge, MA, USA), anti-N-cadherin (ab18203, Abcam), anti-Vimentin (ab92547, Abcam), anti-CBLL1 (ab50993, Abcam), anti-Bcl-2 (ab185002, Abcam), anti-Bax (ab32503, Abcam) and anti-GAPDH (ab181602, Abcam). The secondary antibody was then incubated with the membranes for $2 \mathrm{~h}$ at room temperature. The protein expression was measured by the enhanced chemiluminescence (ECL) system (Millipore).

\section{Lactate dehydrogenase (LDH) cytotoxicity assay}

NSCLC cells were plated into 96-well plates, and LDH releasing reagent $(20 \mu \mathrm{L}$; LDH cytotoxicity assay kit; Beyotime) was added to the wells of 96-well plates. NSCLC cells were incubated with LDH releasing reagent for $1 \mathrm{~h}$ followed by centrifugation for $5 \mathrm{~min}$ at $400 \times g$. The cell supernatant $(120 \mu \mathrm{L})$ of each well was transferred to new 96-well plates, and the optical density was detected at $490 \mathrm{~nm}$.

\section{Dual-luciferase reporter assay}

The miR-545-3p binding sites in circ_0072083 (wile type or mutant type) or the $3^{\prime}$ untranslated region ( $3^{\prime}$ UTR) of CBLL1 (wile type or mutant type) were cloned to pmirGLO vector (Promega, Madison, WI, USA), termed as circ_0072083 WT or circ_0072083 MUT and CBLL1 3' UTR WT or CBLL1 3' UTR MUT. The luciferase activity was analyzed through the dual-Luciferase Reporter Assay System (Promega).

\section{RNA immunoprecipitation (RIP) assay}

Magna RIP RNA-Binding Protein Immunoprecipitation Kit (Millipore) was used in RIP assay to verify the combination between circ_0072083 and miR-545-3p. The NSCLC cells were incubated with protein-A/G Sepharose beads (Bio-Rad, Hercules, CA, USA) pre-coated with Argonaute-2 antibody (Anti-Ago2) or Immunoglobulin $\mathrm{G}$ antibody (Anti-IgG). The complexes were pulled-down 
by the protein-A/G Sepharose beads, and qRT-PCR was carried out to do the following detection.

\section{Animal study}

Nude mice purchased from Laboratory Animal Center of the Academy of Military Medical Sciences (Beijing, China) were inoculated with H522 cells stably expressing Sh-circ_0072083. $3 \mathrm{mg} / \mathrm{kg}$ DDP or phosphate buffered saline (PBS) was intravenously injected into the mice every 5 days after inoculation for 5 days. The size of tumors was measured every 5 days. The nude mice were sacrificed and the tumors were resected after inoculation for 30 days. And then the tumors were weighed, and the enrichment of circ_0072083, miR-545-3p and CBLL1 was detected by qRT-PCR and Western blot. The animal study was performed with the permission of the Institutional Animal Care and Use Committee at First Affiliated Hospital of Henan University.

\section{Statistical analysis}

Data were presented as mean \pm standard deviation (SD). Student's $t$ test or one-way analysis of variance (ANOVA) followed by Tukey's test was used to analyze the differences as appropriate. $P<0.05$ was considered statistically significant.

\section{Results}

Circ_0072083 is aberrantly up-regulated in NSCLC specimens and cells

To determine the role of circ_0072083 in NSCLC, the expression of circ_0072083 in NSCLC tissues and cell lines and their corresponding normal controls was examined. As depicted in Fig. 1a, b, abnormal up-regulation of circ_0072083 was observed in NSCLC tissues and cells relative to normal tissues and normal human lung epithelial cells BEAS-2B. We also explored the subcellular distribution of circ_0072083. Circ_0072083 mainly distributed in the cytoplasmic fraction of $\mathrm{H} 522$ and A549
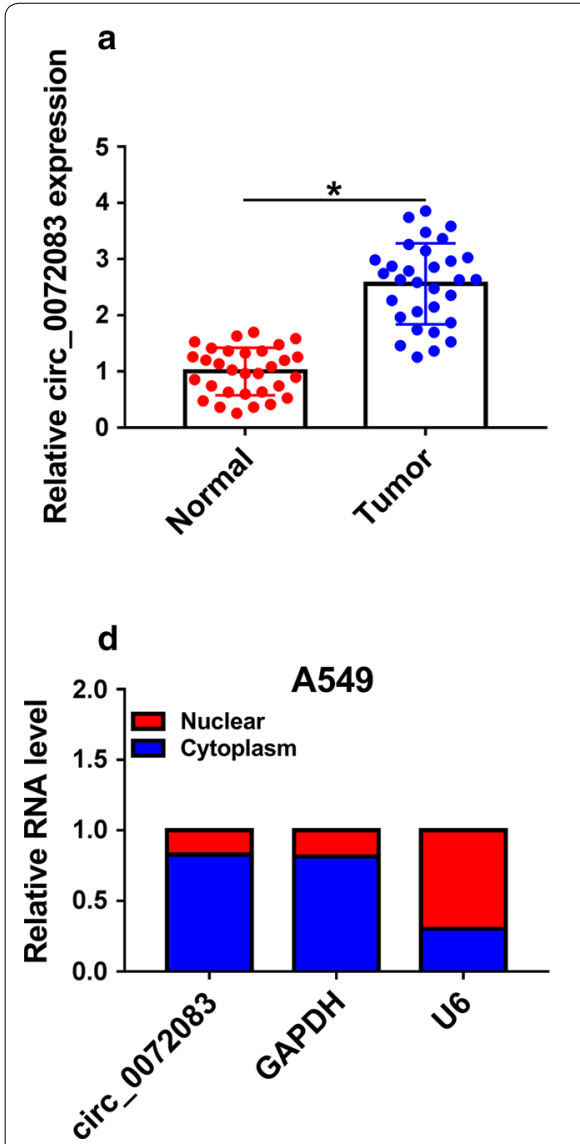
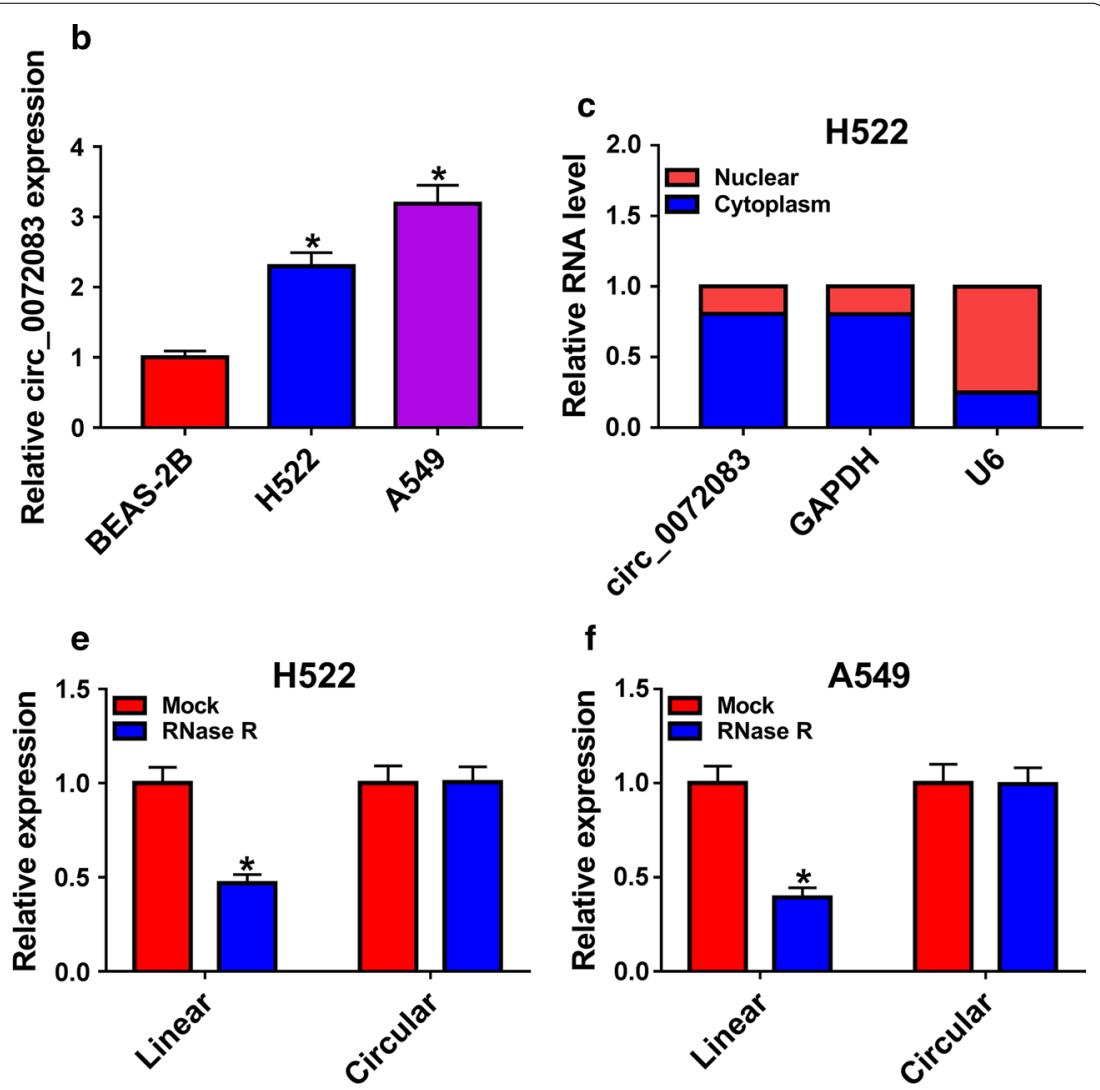

Fig. 1 Circ_0072083 is aberrantly up-regulated in NSCLC specimens and cells. a Expression level of circ_0072083 was detected in NSCLC samples and adjacent normal tissues by qRT-PCR. $\mathbf{b}$ qRT-PCR was performed to measure the expression of circ_0072083 in normal human lung epithelial cell line BEAS-2B and NSCLC cell lines (H522 and A549). c, d The distribution of circ_0072083 in the nuclear or cytoplasm fraction of NSCLC cells was determined by qRT-PCR. e, f The stability of circ_0072083 was assessed in the control group and RNase R group of A549 and H522 cells by qRT-PCR. ${ }^{*} P<0.05$ 
cells (Fig. 1c, d). The stability of circ_0072083 was measured in NSCLC cells treated with RNase R. Compared with matching linear messenger RNA (mRNA; ZFR), circ_0072083 was more stable owing to its closed loop structure (Fig. 1e, f).

\section{Circ_0072083 knockdown decreases the DDP resistance of NSCLC cells}

To further clarify the function of circ_0072083 in NSCLC, circ_0072083 was silenced in H522 and A549 cells through transfecting si-circ_0072083 into the two cells. There was a significant decrease in the level of circ_0072083 in si-circ_0072083 transfected group (Fig. 2a, b). Next, we examined the effects of circ_0072083 knockdown on the colony formation, apoptosis, cell cycle and metastasis of NSCLC cells exposed to DDP. The capacity of colony formation in NSCLC cells was inhibited with the depletion of circ_0072083, and the capacity was further decreased with the addition of DDP (Fig. 2c). The apoptosis rate of NSCLC cells exhibited a reverse phenomenon to the colony formation (Fig. 2d, e). The changes in the expression of pro-apoptotic protein $\mathrm{Bax}$ and anti-apoptotic protein $\mathrm{Bcl}-2$ revealed that circ_0072083 depletion accelerated the apoptosis, and the co-treatment of si-circ_0072083 and DDP further exacerbated the apoptosis of NSCLC cells (Fig. 2f, g). We also investigated the influence of circ_0072083 silencing on the cell cycle of NSCLC cells according to the cell cycle stage distribution (G0/G1, S, G2/M). As indicated in Fig. 2h, i, there was an up-regulation of the cell percentage at G0/G1 phase, suggesting that circ_0072083 depletion arrested cell cycle at G0/G1 phase. Moreover, the results of transwell migration and invasion assays showed that DDP further aggravated circ_0072083 silencing-mediated inhibition of metastasis in NSCLC cells (Fig. 2j, k). Epithelial-mesenchymal transition (EMT) markers, including E-cadherin, $\mathrm{N}$-cadherin and Vimentin, were detected in $\mathrm{H} 522$ and A549 cells treated with si-NC, si-circ_0072083, DDP + si-NC or DDP + sicirc_0072083. The expression of N-cadherin and Vimentin was decreased with the intervention of circ_0072083, and the introduction of DDP exacerbated the inhibitory effect caused by circ_0072083 inhibition (Fig. 2l, m). The abundance of E-cadherin revealed an opposite trend to
$\mathrm{N}$-cadherin or Vimentin, suggesting that DDP promoted the suppressive influence of circ_0072083 depletion on the metastasis of NSCLC cells. Besides, the results of LDH cytotoxicity assay suggested that DDP promoted sicirc_0072083-mediated necrosis of NSCLC cells (Additional file 1: Figure S1). The knockdown of circ_0072083 had no significant effects on the colony formation and apoptosis of normal human lung epithelial cells BEAS-2B (Additional file 2: Figure S2).

\section{Circ_0072083 could modulate the expression of miR-545-3p through directly binding to miR-545-3p}

According to the prediction from starBase online software, there existed binding sites between circ_0072083 and miR-545-3p (Fig. 3a). The results of dual-luciferase reporter assay revealed that the co-transfection of miR545-3p and circ_0072083 WT remarkably declined the luciferase activity, while it remained unchanged in miR545-3p and circ_0072083 MUT group (Fig. 3b, c). RIP experiment suggested that both circ_0072083 and miR545-3p existed in RNA induced silencing complex (RISC) (Fig. 3d, e).

Next, we found that miR-545-3p was down-regulated in NSCLC tissues compared with that in adjacent normal tissues (Fig. 3f). Meanwhile, the low expression of miR-545-3p was also observed in the two NSCLC cell lines in comparison with that in BEAS-2B cells (Fig. 3g). To explore the role of circ_0072083 on the expression of miR-545-3p in NSCLC cells, circ_0072083 siRNA was transfected into NSCLC cells. As shown in Fig. 3h, $\mathrm{i}$, miR-545-3p was up-regulated with the depletion of circ_0072083 in NSCLC cells.

\section{MiR-545-3p depletion overturns the effects of circ_0072083 knockdown on NSCLC cells treated with DDP}

To illustrate whether circ_0072083 exerted its role through sponging miR-545-3p in NSCLC cells, we conducted rescue experiments. As exhibited in Fig. 4a, b, circ_0072083 knockdown increased the abundance of miR-545-3p in NSCLC cells, and this promoting impact was reversed by the addition of anti-miR-545-3p. Further investigation showed that the inhibitory effects on the colony formation, cell cycle and metastasis along

\footnotetext{
(See figure on next page.)

Fig. 2 Circ_0072083 knockdown decreases the DDP resistance of NSCLC cells. a, b The level of circ_0072083 was detected in H522 and A549 cells transfected with si-NC or si-circ_0072083 by qRT-PCR. c-m H522 and A549 cells were treated with si-NC, si-circ_0072083, DDP + si-NC or DDP + si-circ_0072083. c The colony formation ability was detected in NSCLC cells through colony formation assay. d, e The apoptosis rate of NSCLC cells was evaluated by flow cytometry. $\mathbf{f}, \mathbf{g}$ Western blot assay was carried out to detect the apoptosis-related markers in NSCLC cells. $\mathbf{h}, \mathbf{i}$ Cell cycle of NSCLC cells was analyzed by flow cytometry. $\mathbf{j}$, $\mathbf{k}$ The motility of NSCLC cells was detected through conducting transwell migration and invasion assays. I, $\mathbf{m}$ Western blot assay was performed to detect the protein expression of E-cadherin, $\mathrm{N}$-cadherin and Vimentin in NSCLC cells, and GAPDH served as the internal reference in this study. ${ }^{*} P<0.05$
} 


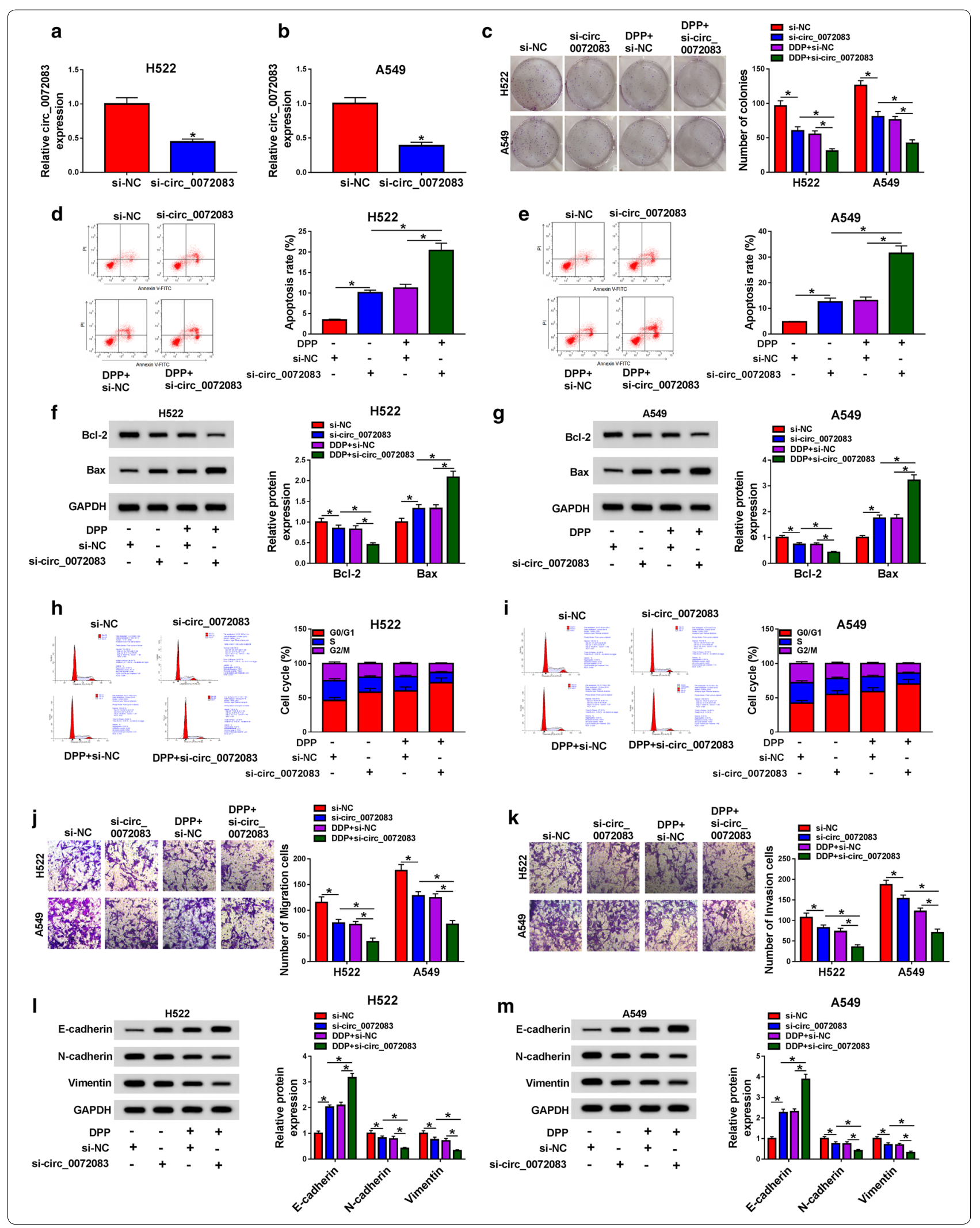




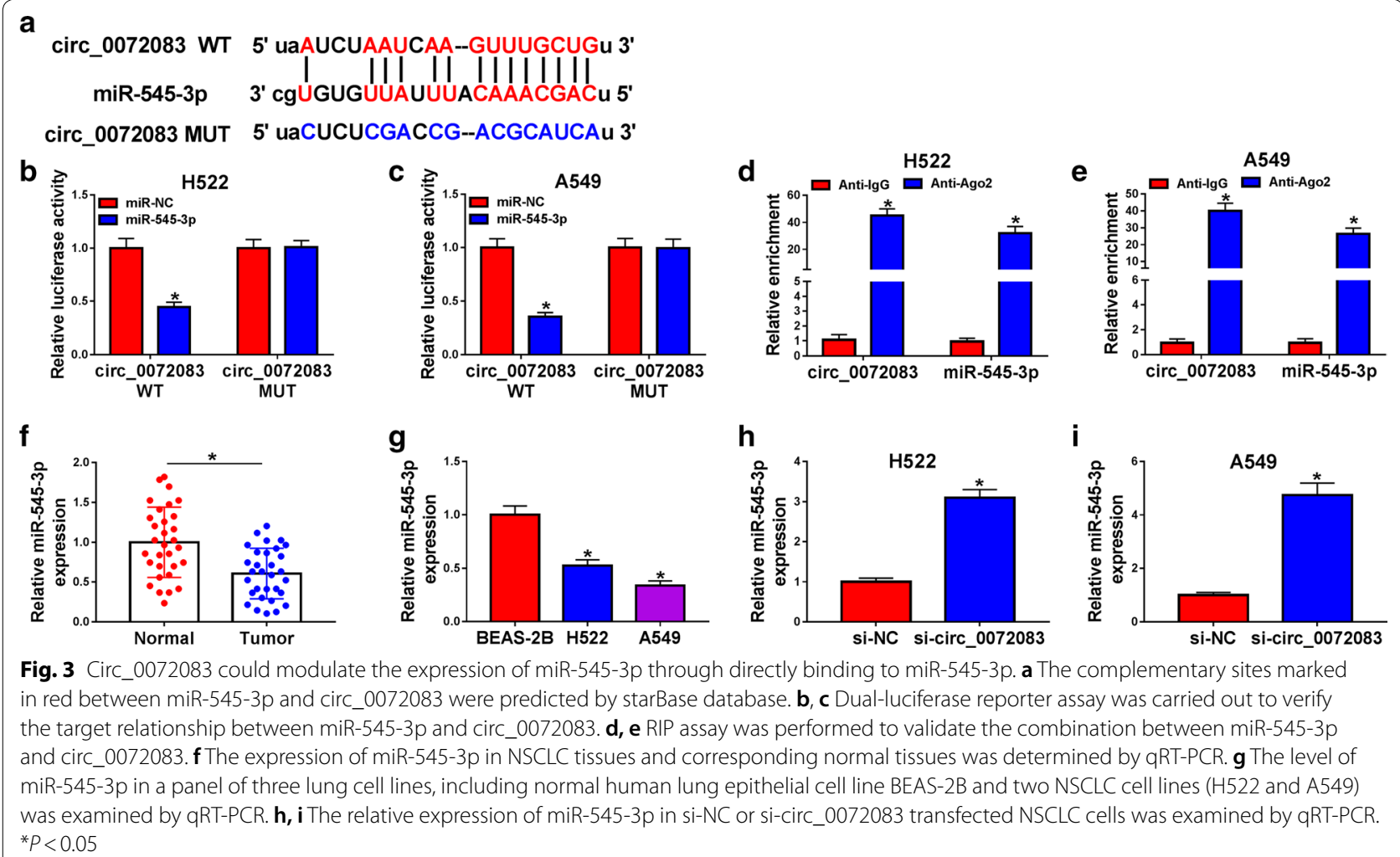

with the promoting influence on the apoptosis of DDPinduced NSCLC cells caused by circ_0072083 interference were overturned by the co-transfection of si-circ_0072083+anti-miR-545-3p (Fig. 4c-n). Besides, western blot assay revealed that the addition of antimiR-545-3p recovered the ability of metastasis, which was suppressed by the intervention of circ_0072083 in DDP-stimulated NSCLC cells (Fig. 4o, p). Furthermore, the addition of anti-miR-545-3p recovered the viability of NSCLC cells stimulated by DDP which was restrained by the interference of circ_0072083 (Additional file 1: Figure S1b).

\section{CBLL1 binds to miR-545-3p in NSCLC cells}

Targetscan software predicted that CBLL1 might be a target of miR-545-3p (Fig. 5a). To validate this prediction, dual-luciferase reporter assay was carried out. As depicted in Fig. 5b, c, miR-545-3p transfection declined the luciferase activity in CBLL1 $3^{\prime}$ UTR WT group other than CBLL1 $3^{\prime}$ UTR MUT group.

There was a higher abundance of CBLL1 in NSCLC tissues relative to matching non-tumor tissues (Fig. 5d). Also, the expression of CBLL1 was higher in NSCLC cells than that in BEAS-2B cells (Fig. 5e). MiR-545-3p mimic and anti-miR-545-3p were transfected into NSCLC cells, and Western blot was performed to detect the protein expression of CBLL1 in the two NSCLC cells. MiR-545-3p accumulation caused a decrease in the expression of CBLL1 in NSCLC cells, while the transfection of anti-miR-545-3p up-regulated the expression of CBLL1 (Fig. 5f, g). As mentioned above, miR-545-3p was a direct target of circ_0072083 in NSCLC cells, and we wondered whether CBLL1 was modulated by circ_007208/miR-545-3p axis. The enrichment of CBLL1 was declined by the transfection of si-circ_0072083 in NSCLC cells, and the level of CBLL1 was regained with the introduction of anti-miR-545-3p (Fig. 5h, i).

MiR-545-3p inhibits the malignant potential of NSCLC cells stimulated with DDP via inhibiting CBLL1

We wondered whether miR-545-3p played an antitumor role through targeting CBLL1 in NSCLC. CBLL1 overexpression recovered the abundance of CBLL1 in NSCLC cells, which was decreased by the transfection of miR-545-3p mimic (Fig. 6a, b). Functional experiments showed that the accumulation of miR-545-3p caused significant inhibition on the colony formation, cell cycle and metastasis along with a marked promotion on the apoptosis of NSCLC cells treated with DDP (Fig. $6 \mathrm{c}-\mathrm{n}$ ), and the co-transfection of CBLL1 and miR$545-3 p$ recovered the malignant potential of NSCLC cells. The changes in the expression of metastasis-related 


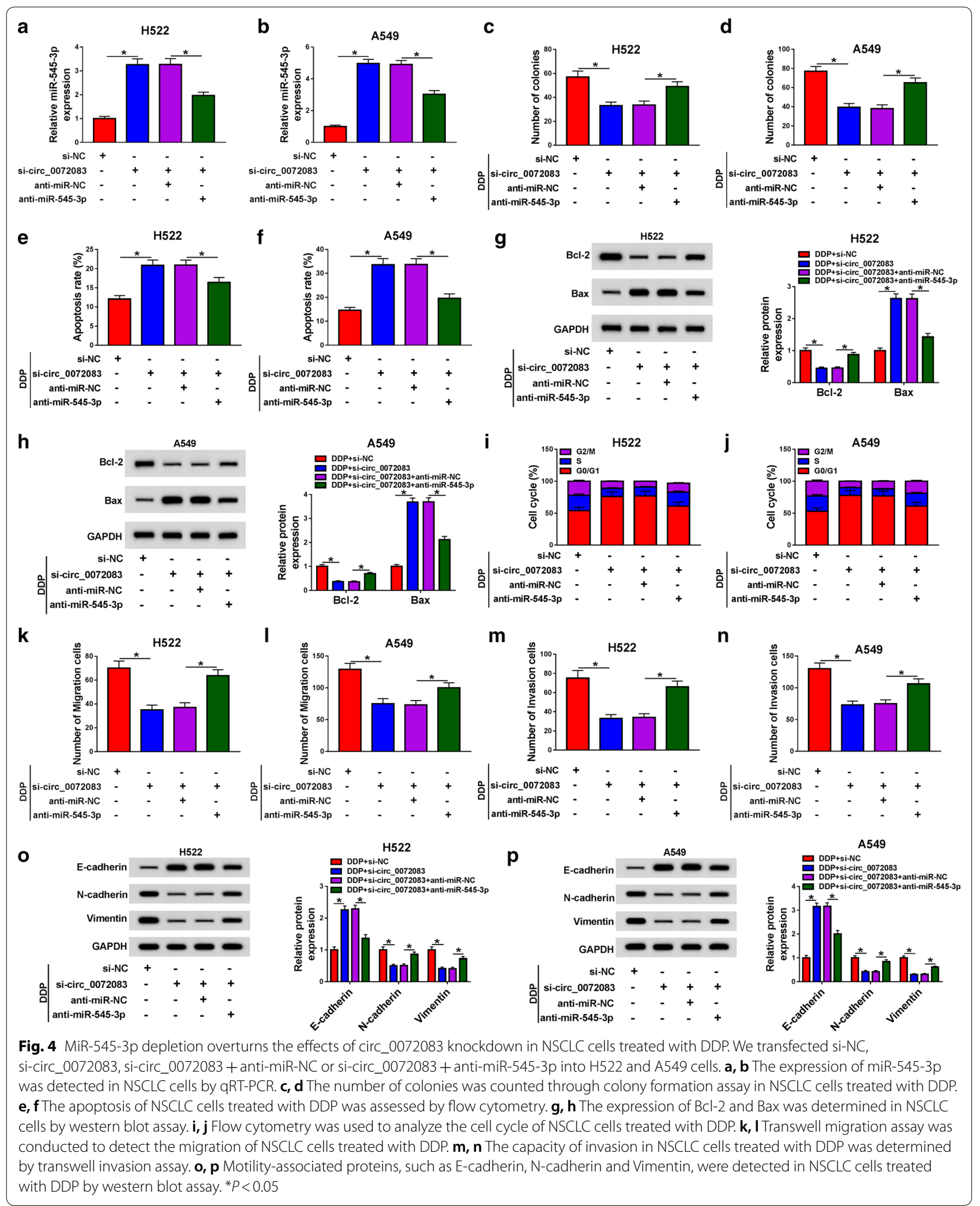




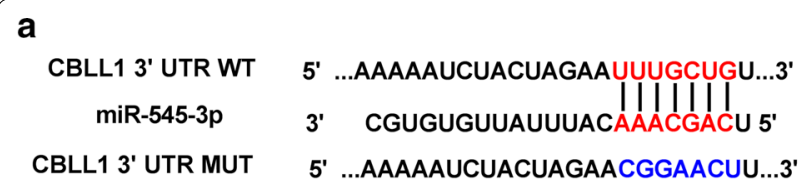

b

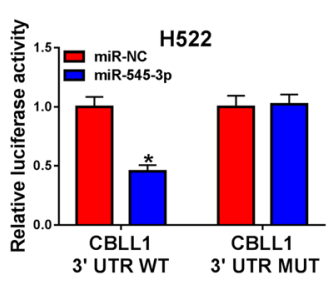

$\mathbf{f}$

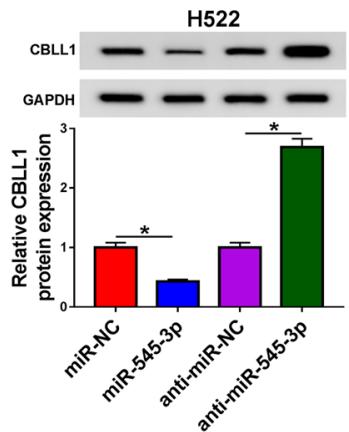

C

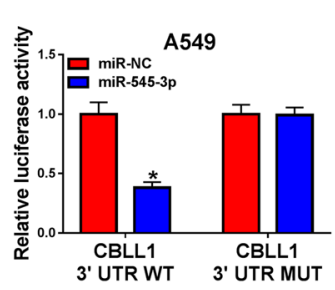

g

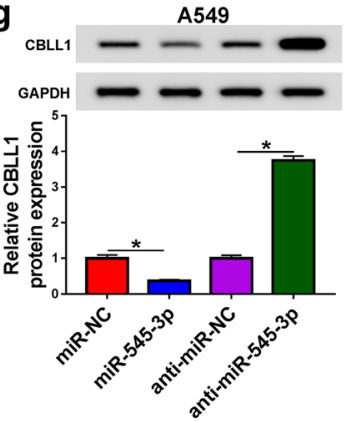

d
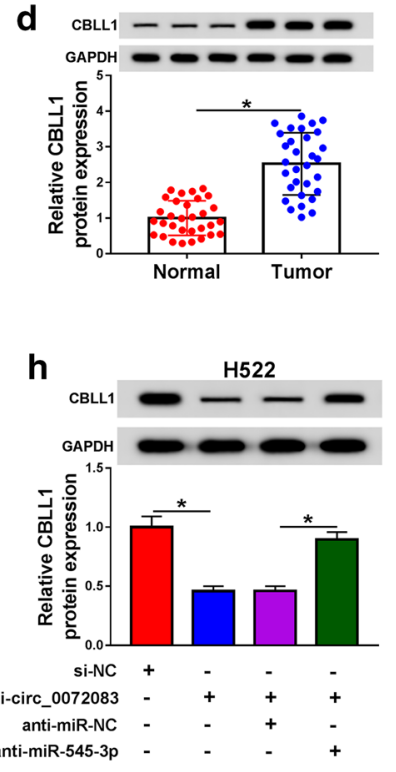

e
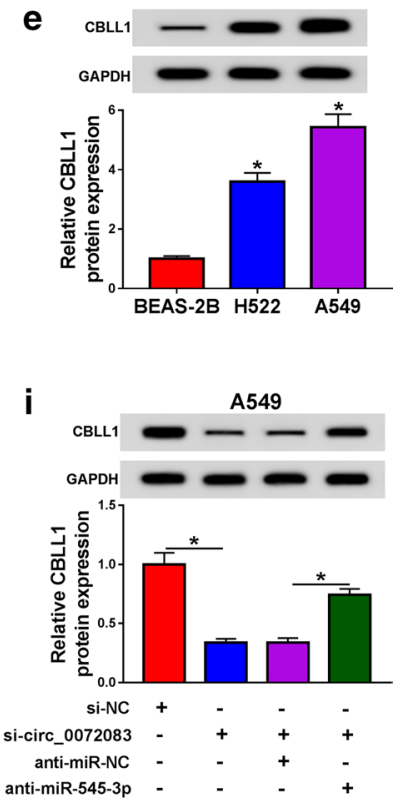

Fig. 5 CBLL1 binds to miR-545-3p in NSCLC cells. a The 3' UTR of CBLL1 possessed the complementary bases with miR-545-3p using Targetscan software. b, c Luciferase activity was determined in H522 and A549 cells co-transfected with miR-NC or miR-545-3p and CBLL1 3' UTR WT or CBLL1 $3^{\prime}$ UTR MUT reporter plasmid. d The protein expression of CBLL1 was examined in NSCLC samples and matching non-tumor tissues by western blot assay. e The level of CBLL1 was measured in BEAS-2B, H522 and A549 cells by western blot assay. $\mathbf{f}, \mathbf{g}$ H522 and A549 cells were transfected with miR-NC, miR-545-3p, anti-miR-NC or anti-miR-545-3p, and Western blot was performed to detect the level of CBLL1 in the above NSCLC cells. $\mathbf{h}$, i The expression of CBLL1 was detected in NSCLC cells transfected with si-NC, si-circ_0072083, si-circ_0072083 + anti-miR-NC or si-circ_0072083 + anti-miR-545-3p by western blot assay. ${ }^{*} P<0.05$

proteins (E-cadherin, N-cadherin and Vimentin) also suggested that miR-545-3p repressed the metastasis of DDP-triggered NSCLC cells through down-regulating CBLL1 (Fig. 6o, p). Moreover, miR-545-3p accumulation promoted the necrosis of NSCLC cells treated with DDP, and the addition of CBLL1 regained the viability of NSCLC cells (Additional file 1: Figure S1c).

\section{Circ_0072083 depletion further aggravates DDP-mediated suppression of NSCLC tumor growth in vivo}

We further tested the effects of circ_0072083 and DDP in xenograft tumor mice. The NSCLC tumors formed in DDP-treated group were lesser than those in the control group (Fig. 7a, b). Furthermore, the depletion of circ_0072083 in NSCLC tumors caused a further decrease in the size and weight of NSCLC tumors. The enrichment of circ_0072083, miR-545-3p and CBLL1 was measured in xenograft tumors. As mentioned in Fig. 7c-e, the abundance of circ_0072083 and CBLL1 was reduced in Sh-circ_0072083 + DDP group compared with that in Control +DDP group, while the expression of miR-545-3p revealed a reverse phenomenon.

\section{Discussion}

CircRNAs have been reported to be associated with the progression of cancers $[20,21]$. The abnormal expression of circRNAs was related to the development of NSCLC. For example, Wang et al. reported that circ_0067934 depletion blocked the growth and motility of NSCLC cells [22]. Li et al. proved that the high expression of circ_0016760 promoted the progression of NSCLC via miR-1287/GAGE1 signaling [23]. Liu et al. found that circ_0001649 repressed the progression of NSCLC through miR-331-3p and miR-338-5p [24]. This study showed that circ_0072083 was highly expressed in NSCLC tissues and cells. Besides, the silencing of circ_0072083 aggravated DDP-induced inhibition of colony formation, cell cycle and metastasis and DDP-mediated up-regulation of apoptosis and necrosis of NSCLC cells. The pro-cancer role of circ_0072083 in NSCLC was in alignment with the former article [12]. 


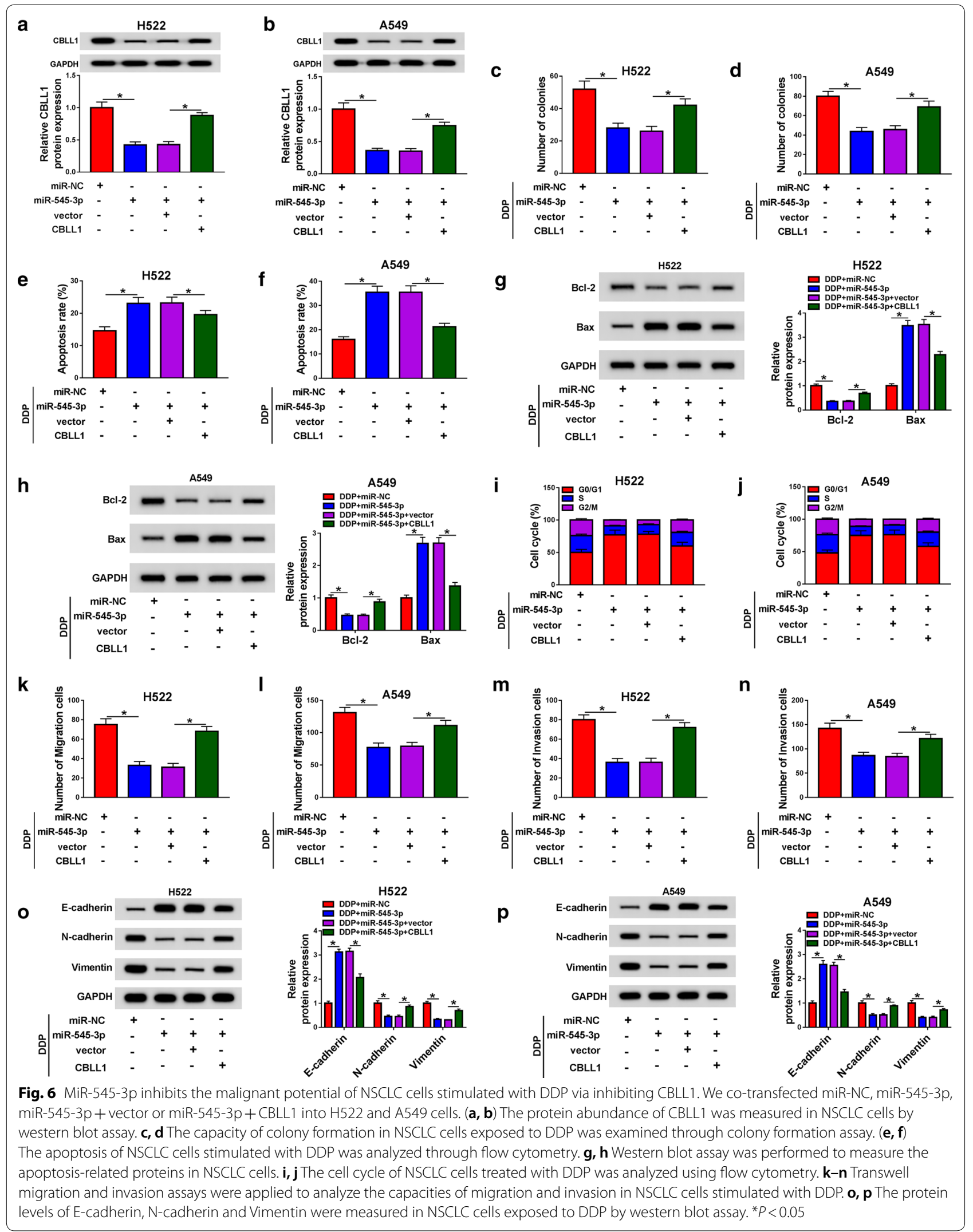




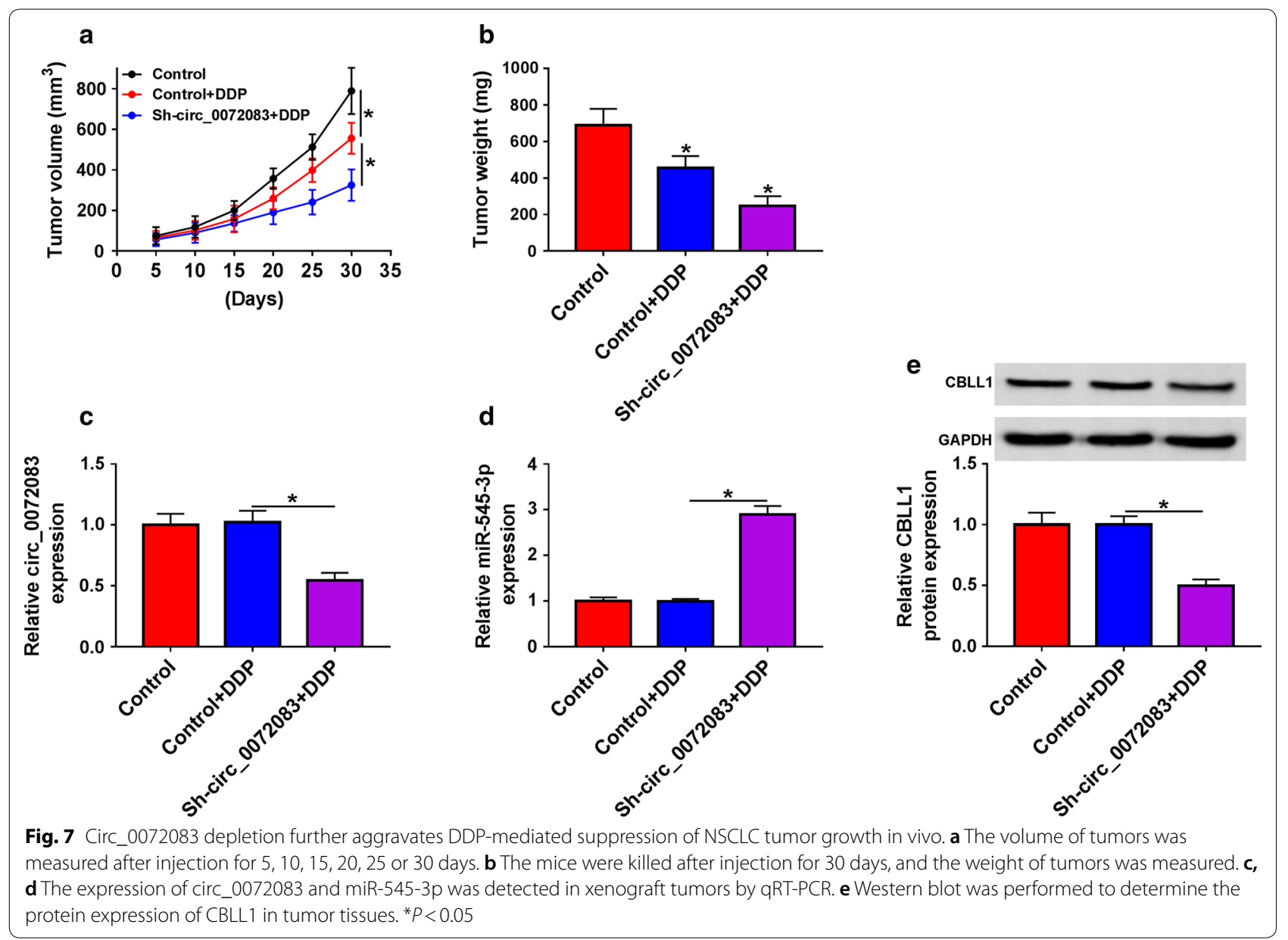

CircRNAs could bind to miRNAs and function as miRNAs sponges [20]. Accumulating articles have reported the downstream miRNAs of circ_0072083 in diverse cancers [25-27]. Herein, miR-545-3p was verified as a target of circ_0072083 in NSCLC cells. MiR-545-3p was an anti-cancer gene in multiple cancers. Chang et al. proved that miR-545-3p inhibited the proliferation, migration and invasion of hepatocellular carcinoma cells via MT1M [28]. Song et al. found that miR-545 hampered the growth of pancreatic ductal adenocarcinoma cells via RIG-1 [29]. In this study, we found that circ_0072083 promoted the progression of DDP-treated NSCLC cells via negatively regulating miR-545-3p.

Next, CBLL1 was predicted and confirmed as a target of miR-545-3p through Targetscan software and dual-luciferase reporter assay, respectively. CBLL1 was up-regulated in NSCLC and breast cancer [18, 19, 30]. Consistent with the previous studies, we found that the expression of CBLL1 was elevated in NSCLC tissues and cells. Besides, CBLL1 served as the downstream gene of circ_0072083/miR-545-3p to protect NSCLC cells against DDP-induced damage. The role of CBLL1 was also confirmed through establishing murine xenograft model in vivo.

\section{Conclusion}

Collectively, circ_0072083 was found to be an oncogene in NSCLC, and circ_0072083 protected NSCLC cells against DDP-triggered injury through miR-545-3p/ CBLL1 axis (Additional file 1: Figure S3).

\section{Supplementary information}

Supplementary information accompanies this paper at https://doi. org/10.1186/s12935-020-1162-x.

Additional file 1: Figure S1. The influence of DDP, circ_0072083, miR-545-3p and CBLL1 on the necrosis of NSCLC cells. a The viability of NSCLC cells treated with si-NC, si-circ 0072083, DDP + si-NC or DDP + sicirc_0072083 was detected by LDH cytotoxicity assay kit. b LDH cytotoxicity assay kit was used to measure the necrosis of DDP-induced NSCLC cells transfected with si-NC, si-circ_0072083, si-circ_0072083 + anti-miR-NC or si-circ_0072083 + anti-miR-545-3p. c The viability of DDP-treated NSCLC cells transfected with miR-NC, miR-545-3p, miR-545-3p + vector or miR$545-3 p+C B L L 1$ was determined through using LDH cytotoxicity assay kit. ${ }^{*} P<0.05$. 
Additional file 2: Figure S2. Circ_0072083 intervention has no significant effects on the colony formation and apoptosis of NSCLC cells. a The knockdown efficiency of si-circ_0072083 in BEAS-2B cells was examined by qRT-PCR. $\mathbf{b}$ Colony formation assay was employed to assess the colony formation ability of NSCLC cells transfected with si-NC or si-circ_0072083. c The apoptosis rate of NSCLC cells transfected with si-NC or sicirc_0072083 was evaluated by flow cytometry. ${ }^{*} P<0.05$.

Additional file 3: Figure S3. Circ_0072083 interference enhances the inhibitory effect of cisplatin on the malignance of NSCLC cells via miR545-3p/CBLL1 axis.

\section{Abbreviations}

NSCLC: Non-small-cell lung cancer; qRT-PCR: Quantitative real time polymerase chain reaction; ZFR: Zinc finger RNA binding protein; CBLL1: Cbl protooncogene like 1; RIP: RNA immunoprecipitation.

\section{Acknowledgements}

None.

\section{Authors' contributions}

HL participated in the design of the work, methodology, data interpretation, and analysis for the work, carried out the statistical analysis and drafted the manuscript. FL participated in the methodology, data interpretation, and analysis for the work. WQ designed the study, participated in data interpretation and methodology. All authors read and approved the final manuscript.

\section{Funding}

This work was supported by National Natural Science Foundation, The value of combined application of proteomic MRM-MS and genomic HTS-ctDNA on individualized treatment and healing evaluation of lung cancer (Grant No. 81572911).

\section{Availability of data and materials}

The data that support the findings of this study are available on request from the corresponding author.

\section{Ethics approval and consent to participate}

Not applicable.

\section{Consent for publication}

Not applicable.

\section{Competing interests}

The authors declare that they have no financial conflicts of interests.

Received: 10 December 2019 Accepted: 4 March 2020

Published online: 12 March 2020

\section{References}

1. Duruisseaux M, Esteller M. Lung cancer epigenetics: from knowledge to applications. Semin Cancer Biol. 2018;51:116-28.

2. Bray F, Ferlay J, Soerjomataram I, Siegel RL, Torre LA, Jemal A. Global cancer statistics 2018: GLOBOCAN estimates of incidence and mortality worldwide for 36 cancers in 185 countries. CA Cancer J Clin. 2018;68:394-424.

3. Tsim S, O'Dowd CA, Milroy R, Davidson S. Staging of non-small cell lung cancer (NSCLC): a review. Respir Med. 2010;104(12):1767-74

4. Watanabe SI, Nakagawa K, Suzuki K, Takamochi K, Ito H, Okami J, Aokage K, Saji H, Yoshioka H, Zenke Y, et al. Neoadjuvant and adjuvant therapy for Stage III non-small cell lung cancer. Jpn J Clin Oncol. 2017;47(12):1112-8.

5. Ebbesen KK, Kjems J, Hansen TB. Circular RNAs: identification, biogenesis and function. Biochim Biophys Acta. 2016;1859(1):163-8.

6. Meng S, Zhou H, Feng Z, Xu Z, Tang Y, Li P, Wu M. CircRNA: functions and properties of a novel potential biomarker for cancer. Mol Cancer. 2017;16(1):94.
7. Zhang HD, Jiang LH, Sun DW, Hou JC, Ji ZL. CircRNA: a novel type of biomarker for cancer. Breast Cancer. 2018;25(1):1-7.

8. Kristensen LS, Hansen TB, Veno MT, Kjems J. Circular RNAs in cancer: opportunities and challenges in the field. Oncogene. 2018;37(5):555-65.

9. Wei H, Pan L, Tao D, Li R. Circular RNA circZFR contributes to papillary thyroid cancer cell proliferation and invasion by sponging miR-1261 and facilitating C8orf4 expression. Biochem Biophys Res Commun. 2018;503(1):56-61.

10. Tan A, Li Q, Chen L. CircZFR promotes hepatocellular carcinoma progression through regulating miR-3619-5p/CTNNB1 axis and activating Wnt/beta-catenin pathway. Arch Biochem Biophys. 2019;661:196-202.

11. Wang M, Gao Y, Liu J. Silencing circZFR inhibits the proliferation, migration and invasion of human renal carcinoma cells by regulating miR-206. Onco Targets Ther. 2019;12:7537-50.

12. Zhang $H$, Wang $X$, Hu B, Zhang F, Wei H, Li L. Circular RNA ZFR accelerates non-small cell lung cancer progression by acting as a miR-101-3p sponge to enhance CUL4B expression. Artif Cells Nanomed Biotechnol. 2019;47(1):3410-6.

13. Muhammad N, Bhattacharya S, Steele R, Ray RB. Anti-miR-203 suppresses ER-positive breast cancer growth and stemness by targeting SOCS3. Oncotarget. 2016;7:58595-605.

14. Fan Z, Cui H, Xu X, Lin Z, Zhang X, Kang L, Han B, Meng J, Yan Z, Yan X, Jiao S. MiR-125a suppresses tumor growth, invasion and metastasis in cervical cancer by targeting STAT3. Oncotarget. 2015;6:25266-80.

15. Du M, Zhang Y, Mao Y, Mou J, Zhao J, Xue Q, Wang D, Huang J, Gao S, Gao Y. MiR-33a suppresses proliferation of NSCLC cells via targeting METTL3 mRNA. Biochem Biophys Res Commun. 2017;482(4):582-9.

16. Wang P, Lv HY, Zhou DM, Zhang EN. miR-204 suppresses non-small-cell lung carcinoma (NSCLC) invasion and migration by targeting JAK2. Genet Mol Res. 2016;15(2):1-9.

17. Cui J, Pan G, He Q, Yin L, Guo R, Bi H. MicroRNA-545 targets ZEB2 to inhibit the development of non-small cell lung cancer by inactivating Wnt/beta-catenin pathway. Oncol Lett. 2019;18(3):2931-8.

18. Hui L, Zhang S, Wudu M, Ren H, Xu Y, Zhang Q, Qiu X. CBLL1 is highly expressed in non-small cell lung cancer and promotes cell proliferation and invasion. Thorac Cancer. 2019;10(6):1479-88.

19. Qiu HB, Yang K, Yu HY, Liu M. Downregulation of long non-coding RNA XIST inhibits cell proliferation, migration, invasion and EMT by regulating miR-212-3p/CBLL1 axis in non-small cell lung cancer cells. Eur Rev Med Pharmacol Sci. 2019;23(19):8391-402.

20. Zhu LP, He YJ, Hou JC, Chen X, Zhou SY, Yang SJ, Li J, Zhang HD, $\mathrm{Hu} J \mathrm{H}$, Zhong SL, et al. The role of circRNAs in cancers. Biosci Rep. 2017;37(5):BSR20170750. https://doi.org/10.1042/BSR20170750.

21. Qian L, Yu S, Chen Z, Meng Z, Huang S, Wang P. The emerging role of circRNAs and their clinical significance in human cancers. Biochim Biophys Acta Rev Cancer. 2018;1870(2):247-60.

22. Wang J, Li H. CircRNA circ_0067934 silencing inhibits the proliferation, migration and invasion of NSCLC cells and correlates with unfavorable prognosis in NSCLC. Eur Rev Med Pharmacol Sci. 2018;22(10):3053-60.

23. Li Y, Hu J, Li L, Cai S, Zhang H, Zhu X, Guan G, Dong X. Upregulated circular RNA circ_0016760 indicates unfavorable prognosis in NSCLC and promotes cell progression through miR-1287/GAGE1 axis. Biochem Biophys Res Commun. 2018;503(3):2089-94.

24. Liu T, Song Z, Gai Y. Circular RNA circ_0001649 acts as a prognostic biomarker and inhibits NSCLC progression via sponging miR-331-3p and miR-338-5p. Biochem Biophys Res Commun. 2018;503(3):1503-9.

25. Yang X, Liu L, Zou H, Zheng YW, Wang KP. circZFR promotes cell proliferation and migration by regulating miR-511/AKT1 axis in hepatocellular carcinoma. Dig Liver Dis. 2019;51(10):1446-55.

26. Zhang WY, Liu QH, Wang TJ, Zhao J, Cheng XH, Wang JS. CircZFR serves as a prognostic marker to promote bladder cancer progression by regulating miR-377/ZEB2 signaling. Biosci Rep. 2019;39(12):BSR20192779. https://doi.org/10.1042/BSR20192779.

27. Liu T, Liu S, Xu Y, Shu R, Wang F, Chen C, Zeng Y, Luo H. Circular RNAZFR inhibited cell proliferation and promoted apoptosis in gastric cancer by sponging miR-130a/miR-107 and modulating PTEN. Cancer Res Treat. 2018;50(4):1396-417.

28. Changjun L, Feizhou H, Dezhen P, Zhao L, Xianhai M. MiR-545-3p/ MT1M axis regulates cell proliferation, invasion and migration in hepatocellular carcinoma. Biomed Pharmacother. 2018;108:347-54. 
29. Song B, Ji W, Guo S, Liu A, Jing W, Shao C, Li G, Jin G. miR-545 inhibited pancreatic ductal adenocarcinoma growth by targeting RIG-I. FEBS Lett. 2014;588(23):4375-81.

30. Gong EY, Park E, Lee K. Hakai acts as a coregulator of estrogen receptor alpha in breast cancer cells. Cancer Sci. 2010;101(9):2019-25.

\section{Publisher's Note}

Springer Nature remains neutral with regard to jurisdictional claims in published maps and institutional affiliations.
Ready to submit your research? Choose BMC and benefit from:

- fast, convenient online submission

- thorough peer review by experienced researchers in your field

- rapid publication on acceptance

- support for research data, including large and complex data types

- gold Open Access which fosters wider collaboration and increased citations

- maximum visibility for your research: over 100M website views per year

At BMC, research is always in progress.

Learn more biomedcentral.com/submissions 